\title{
EDITORIAL
}

\section{Is the risk of acute lymphoblastic leukemia reduced in siblings to children with the disease? A novel hypothesis explored by international collaboration}

Leukemia (2006) 20, 1206-1208. doi:10.1038/sj.leu.2404250

In spite of all the epidemiological data that associate the risk of acute lymphoblastic leukemia (ALL) to genetic predisposition and environmental exposures, the incidence of childhood ALL among siblings to children with the disease is at most only weakly increased, and a large population- and registerbased study indicates that it may even be reduced. ${ }^{1}$ We here summarize these data that have led to an international collaboration, and a call for colleagues, who in their career have encountered families with two or more cases of childhood ALL in a sibship. Physicians who in their career have encountered such a family are asked to register some basic data on a one-page data sheet, which can be provided for, on request to kschmiegelow@rh.dk.

For the majority of childhood cancers, incidence peaks early in childhood or even during fetal life. Chemotherapy is typically more effective for the malignancies that occur in these age peak groups than for those that occur later in childhood as seen for neuroblastoma (peak $<1$ year), hepatoblastoma (peak $<1$ year), rhabdomyosarcoma (peak 1-7 years), ALL (peak 2-7 years) and Wilms' tumor (peak 2-6 years). ${ }^{2}$ Furthermore, spontaneous regression is well known for infant neuroblastoma, but has also been described for other entities such as GATA1mutated neonatal leukemia of Down's syndrome, and some other rare congenital myeloid leukemias. For some of the chidlhood malignancies, recent evidence suggest that the proportion of children that harbor premalignant tumors or hemopoietic cells with leukemia-associated cytogenetic aberrations at birth by far exceeds the proportion of children who develop overt cancer, for example, by several folds for neuroblastoma and by at least 100 -fold for childhood ALL. ${ }^{3}$

These characteristics raise the question whether some childhood cancers may develop if certain natural mechanisms fail to control premalignant cells that develop in utero or during the first years of life. The confirmation and exploration of such (hypothetical) mechanisms would be of interest to the understanding of the natural history of childhood cancer, but more importantly could also lead to preventive measures through enhancement or mimicking of these mechanisms. In this respect, childhood ALL could serve as the proof of principle.

Most cases of childhood ALL occur in the age group of 2-7 years, and the incidence peak reaches a maximum at 3 years, where it may be 10 times higher than in older and younger children. ${ }^{4}$ The majority of cases in the incidence peak are characterized by a high-hyperdiploid clone (typically trisomies $X, 4,6,10,14,17,18$ and 21) or a translocation $\mathrm{t}(12 ; 21)$ (p13;q22) [ETV6/RUNX1]. A prenatal origin of the first cytogenetic aberration(s) in t(12;21)-positive, high-hyperdiploid and some other subsets of ALL has been suggested by mathematical modelling of the incidence curve, and demonstrated in twin studies, and by backtracking of clone-specific cytogenetic aberrations or immune gene rearrangements to the Guthrie cards from children with ALL. ${ }^{5-8}$
However, whereas approximately 1:10000 of all children develops $\mathrm{t}(12 ; 21)$-positive $\mathrm{ALL}$, studies suggest that at least $1: 100$ healthy individuals harbour $\mathrm{t}(12 ; 21)$-positive cells in their peripheral blood at a level of $10^{-3}-10^{-4}$. $^{3}$ The $t(12 ; 21)$ translocations are functional, but may be balanced by the normal TEL/ETV6 gene. ${ }^{9}$ The occurrence and possibly also the timing of subsequent, most likely postnatal, genetic aberrations probably determine, whether a child that carry t(12;21)-positive preleukemic cells will develop ALL. ${ }^{8-10}$

Exploration of the prenatal origin of childhood leukemia and the significance of the genetic aberrations is critical for the understanding of the natural history of childhood ALL. High birth weight, low gestational age and certain in utero exposures have all been related to the risk of ALL, but it is not known whether they exert their effects by being risk factors for the development of preleukemic cells in an otherwise healthy fetus. ${ }^{8,10,11}$ Similarly, the postnatal exposures that eventually may induce ALL in children harboring preleukemic cells remain to be determined, but among others they seem to correlate with high socioeconomic standards, including a delay in infectious exposures in early childhood (for a review see McNally and Eden ${ }^{12}$ ). This has been supported by studies of the incidence of ALL (and phenotypic subtypes) in countries with high versus low socioeconomic status, cluster analyses, studies of population mixing, mapping of infection patterns in case-control studies and by genetic studies that have linked the risk of ALL to certain alleles of polymorphic genes that affect responses to infections. ${ }^{13,14}$ Furthermore, folate intake during pregnancy and genetic polymorphisms that may affect folate metabolism (and thus DNA-synthesis and -repair) have been implicated in the development of ALL. ${ }^{15}$ The significance of the right environmental and genetic setting is further illustrated by twin studies that indicate that if preleukemic cells are present at birth, the risk of childhood ALL may be as high as $5-10 \%{ }^{8}$

As a large fraction of healthy newborns may carry preleukemic cells, and as many studies have indicated that both genetic and environmental factors influence the risk of $A L L$, an increased occurrence of ALL among siblings to children with ALL would be expected. Consistent with this, two large studies from the United States and from England with exploration of 37000 and 51000 death certificates, respectively, indicated a twofold increased risk of leukemia among siblings to children with leukemia. ${ }^{16,17}$ However, the studies did not distinguish between types of leukemia, and it is uncertain to what extent other severe bone marrow diseases or leukemoid reactions were misclassified as leukemias on the death certificates. More recently, a population- and register-based study from the Nordic countries revealed only two cases of non-twin leukemia (including one undifferentiated) among approximately 11000 non-twin siblings to 6313 children with leukemia compared to the five cases that were expected by chance. ${ }^{1}$

As the proportion of newborns harboring preleukemic $\mathrm{t}(12 ; 21)$-positive cells probably is at least 100 times the proportion of children developing $\mathrm{t}(12 ; 21)$-positive ALL, the likelihood that such preleukemic cells degenerate further to overt ALL is 
$1 \%$ at the most. One explanation for the low incidence of ALL among siblings could be that, both the pre- and postnatal genetic aberrations that lead to common childhood ALL occur as random events. In that scenario, not only would the risk of any sibling developing ALL be as in the background population, but all the epidemiological findings that link the risk of ALL to genetic predisposition and/or environmental exposures should be regarded as chance phenomena. This explanation seems unlikely given the quantity of these studies. ${ }^{8,10-15}$

Why, then did the recent large population- and register-based Nordic study indicate that the risk of childhood is not increased in siblings to children with ALL and perhaps even reduced? ${ }^{1}$ Both the presence of preleukemic cells at birth and the appropriate genetic predisposition and postnatal environment are believed to be necessary for the development of childhood $\mathrm{ALL}$ in the age peak group. ${ }^{10}$ If for instance, $\mathrm{t}(12 ; 21)$-positive preleukemic cells were unlikely to occur and persist in more than one of a woman's pregnancies, the risk of ALL among the siblings to that child would be reduced to that of the remaining types of prenatally initiated cases (e.g. high-hyperdiploid ALL) plus that of subtypes that are initiated postnatally. Several mechanisms could prevent the occurrence of preleukemic cells in multiple pregnancies.

First, as most cases of childhood ALL are diagnosed in early childhood, the mother may change living habits while still fertile. Thus, the risk of in utero DNA damage could be influenced by changes in dietary factors (e.g. folate status ${ }^{15}$ ) or exposure to carcinogens or to infections during subsequent pregnancies and in early postnatal life (see below). This could reduce the risk of emergence of preleukemic cells as well as their further degeneration to manifest leukemia.

Second, if the emergence of preleukemic cells in utero were caused by specific infections during pregnancy, prenatal maternal immunity against such microorganisms would reduce the risk of ALL in later children. However, no specific infections have been convincingly linked with childhood leukemia. ${ }^{12}$ Thus, while moderately increased risks of childhood ALL have been reported following varicella (nonsignificant), influenza (nonsignificant) or Epstein-Barr infections during pregnancy, other studies have showed that the risk of childhood ALL is associated with infections in general, such as recurrent maternal infections, lower genital tract infections and nonspecific viral infections. ${ }^{12}$ The significant odds ratio in the studies that did indicate an association, ranged between 1.5 and 4.0, most being in the order of 1.5-2.0. As these studies indicate that unspecific infections plays a role, immunisation against specific infections is unlikely to explain a reduced risk of childhood ALL among siblings.

An alternative third explanation could be compatible with both the suspected low incidence among siblings and some of the known risk factors. When a child develops ALL, this reflects a combination of prenatal occurrence of preleukemic cells and subsequent further genetic degeneration occurring randomly or owing to further DNA-damaging exposures. During pregnancy, such preleukemic cells could cross the placenta and induce an immune response directed against leukemia-specific epitopes on the preleukemic cells. Presence of normal fetal cells in the mother is common, not least at the end of pregnancy, ${ }^{18}$ and such cells can trigger an immune response directed against hematopoietic cells. Best known examples of such crossplacental immune reactions are the hemolytic anemias of the newborn owing to blood type incompatability, and more rarely neonatal thrombocytopenia or neutropenia, as well as other organ dysfunctions seen in children of mothers with systemic lupus erythromatosis. ${ }^{19}$ Such immunization will primarily be induced at the end of a pregnancy, when the passage of fetal cells to the mother is increased. ${ }^{18}$ If the immunization is directed against leukemia-associated antigens, similar preleukemic cells (e.g. t(12;21)-positive) could in a subsequent pregnancy be reduced in frequency or even eliminated. Thus, if a subtype of ALL develops in a child as a consequence of a specific combination of genetic susceptibility and environmental factors, then later siblings to this child would a priori have an increased risk of developing that specific subset of ALL. However, if a prerequisite were that preleukemic cells should develop prenatally, the mother's immune system (possibly by transplacentally transferred antibodies) would be able to eliminate the preleukemic cells should they occur. On the other hand, as this mechanism would be ALL subtypespecific, the risk of other subtypes of ALL would be similar to that of the background population. Thus, the overall risk of ALL in a sibling to an older child with ALL would be slightly less than 1.0 (e.g. 0.80 in case of protection against $t(12 ; 21)$-positive ALL). Naturally, this protective mechanism would only be active for those leukemias, where the initiating events take place prenatally.

Epidemiological findings support such a protective mechanism: (i) A low risk of more than one case per sibship of a specific childhood cancer, many of which probably are initiated prenatally, seem to be a more general feature and not just restricted to leukemia. ${ }^{1}$ (ii) The inverse correlation between the risk of ALL and gestational age at birth ${ }^{11}$ could reflect that transplancental passage of antibodies rises significantly in the weeks before term. (iii) The hypothesis is also compatible with the inverse correlation of the risk of ALL and birth order, that has been indicated in some although not all studies, but which was quite strong in a recent very large Nordic populationbased case-control study that included 1905 children with ALL and 10745 population control subjects matched on nationality, age and sex. In that study, significantly reduced risks of B-precursor ALL were observed with increasing position in the birth order (odds ratio $(\mathrm{OR})=0.90$ per position increase). ${ }^{11}$ Owing to the high frequency of preleukemic cells among healthy newborns, high birth order would be related to an increased likelihood of the mother being immunized to one or more subtypes of preleukemic cells during previous pregnancies. In contrast, the relationship to birth order has previously been interpreted as a surrogate marker for enhanced infectious exposures in early childhood.

If the average sibship size is two, and the risk for any child to develop ALL before the age of 15 is 1:2000, only one sibship with two cases of childhood ALL would occur for every 4000 cases of ALL registered. Thus, it would require almost unsurmountable, international collaboration to demonstrate that the OR among the ALL siblings for specific cytogenetic subsets of ALL (specifically translocation t(12;21)-positive and highhyperdiploid ALL) was significantly lower than 1.0. Such a study would be hampered not only by the huge number of families needed, but also by the likelihood of incomplete ascertainment. Alternatively, an international study could focus only on the families with two (or more) cases of childhood ALL. Given the known frequency of the most common cytogenetic aberrations (e.g. high-hyperdiploidy (25-30\% of all cases), t $(12 ; 21)(20 \%)$, $\mathrm{t}(1 ; 19)(1-5 \%), \mathrm{t}(? ; 11)(? ; \mathrm{q} 23)(1-3 \%)$ and $\mathrm{t}(9 ; 22)(2-3 \%))^{2}$ at least six cases of siblings with the same cytogenetic aberrations would be expected to occur among 45 sibships with two cases of $A L L$, if these cases occurred randomly. If no cases of matching cytogenetic aberrations were found, this would differ significantly from the expected number, and thus lend support to the hypothesis. Such an international accrual of ALL sibships has 
now been initiated by the Ponte di Legno group that includes representatives from ALL trial groups in Italy (AIEOP), Germany (BFM and COALL), Holland (DCOG), USA (Dana Farber Cancer Institute, St Jude Children Research Hospital), European Organisation for Research and Treatment of Cancer (EORTC), France (FRALLE), Japan (JACLS), United Kingdom (MRC-UKALL) and the Nordic countries (NOPHO). ${ }^{20}$ In addition to exploring the cytogenetic patterns within ALL sibships, the study will look at the presence of known genetic predisposition (e.g. Recklinghausen neurofibromatosis type 1 or ataxia telangiectasia), the immune phenotype distribution, white blood cell count and age at diagnosis and age and time intervals between the cases in a sibship, that is, concordance in age (diagnosed at same age) and in time (diagnosed within the same calender period). Furthermore, if available, archieval material will be collected to explore further the karyotypes of the malignant clones and of possible inherited genetic disorders that may predispose to ALL. If there were a familial disposition towards the occurrence of preleukemic cells in utero (mostly expected to be a $t(12 ; 21)$ positive or high-hyperdiploid karyotype) and no immune protection, an augmented age peak with an age mode of 3 years would be expected. ${ }^{4}$ If on the other hand, familial predisposition was restricted to the patients, who have other subsets of ALL than these two subtypes (e.g. T-lineage ALL in ataxia telangiectasia), the age distribution peak would be less prominent. Finally, if two or more cases of ALL in a sibship truly occurred randomly, the incidence curve would be as among singletons with ALL. As the hypothesis proposes that specific mechanisms may protect against the occurrence of preleukemic cells in utero, the expected findings are (i) a less pronounced age distribution peak and (ii) a cytogenetic concordance rate that is significantly lower than the expected random concordance (e.g. very few or no cases where both siblings have $t(12 ; 21)$ or both have high-hyperdiploidy).

It is comforting for parents to children with ALL to know that the risk of ALL in their other children is not significantly higher than the risk in the background population. ${ }^{1}$ However, the proposed hypothesis has perspectives for the prevention of ALL. If immunization of mothers to leukemia-specific antigens can enhance elimination of preleukemic cells in utero, development of preventive measures such as vaccination of women toward appropriate epitopes (when identified) could prevent their children from developing ALL. If succesful, it is also possible that this approach could be applied to other typical childhood cancers.

Physicians who in their career have encountered a family with two or more cases of chilhood ALL are asked to register some basic data on a one-page data sheet, which can be provided for on request to kschmiegelow@rh.dk.

\section{Acknowledgements}

This study is supported by a grant from The Otto Christensen Foundation.

K Schmiegelow ${ }^{1}$ and $\mathrm{H} \mathrm{Hjalgrim}^{2}$

${ }^{1}$ Pediatric Clinic, The University Hospital Rigshospitalet, The Serum Institute, Copenhagen, Denmark and ${ }^{2}$ Department of Epidemiological Research, The Serum Institute, Copenhagen, Denmark E-mail: kschmiegelow@rh.dk

\section{References}

1 Winther JF, Sankila R, Boice JD, Tulinius H, Bautz A, Barlow L et al. Cancer in siblings of children with cancer in the Nordic countries: a population-based cohort study. Lancet 2001; 358: 711-717.

2 Voue PA, Barrett A, Stevens MCG, , Caron HN. Cancer in Children. (eds). 5th edn. Oxford University Press: Oxford, 2005.

3 Mori H, Colman SM, Xiao Z, Ford AM, Healy LE, Donaldson C et al. Chromosome translocations and covert leukemic clones are generated during normal fetal development. Proc Natl Acad SCi USA 2002; 99: 8242-8247.

4 Hjalgrim LL, Rostgaard K, Schmiegelow K, Soderhall S, Kolmannskog S, Vettenranta $\mathrm{K}$ et al. Age- and sex-specific incidence of childhood leukemia by immunophenotype in the Nordic countries. J Natl Cancer Inst 2003; 95: 1539-1544.

5 Wiemels JL, Cazzaniga G, Daniotti M, Eden OB, Addison GM, Masera $G$ et al. Prenatal origin of acute lymphoblastic leukaemia in children. Lancet 1999; 354: 1499-1503.

6 Panzer-Grumayer ER, Fasching K, Panzer S, Hettinger K, Schmitt K, Stockler-Ipsiroglu $\mathrm{S}$ et al. Nondisjunction of chromosomes leading to hyperdiploid childhood B-cell precursor acute lymphoblastic leukemia is an early event during leukemogenesis. Blood 2002; 100: $347-349$.

7 Hjalgrim LL, Madsen HO, Melbye M, Jorgensen P, Christiansen M, Andersen MT et al. Presence of clone-specific markers at birth in children with acute lymphoblastic leukaemia. Br J Cancer 2002; 87: 994-999.

8 Greaves M, Maia AT, Wiemels JL, Ford AM. Leukemia in twins: lessons in natural history. Blood 2003; 102: 2321-2333.

9 Zuna J, Ford A, Peham M, Patel N, Saha V, Eckert C et al. TEL deletion analysis supports a novel view of relapse in childhood acute lymphoblastic leukemia. Clin Cancer Res 2004; 10: 5355-5360.

10 Greaves M. Molecular genetics, natural history and the demise of childhood leukaemia. Eur J Cancer 1999; 35: 1941-1953.

11 Hjalgrim LL, Rostgaard K, Hjalgrim H, Westergaard T, Thomassen $\mathrm{H}$, Forestier $\mathrm{E}$ et al. Birth weight as a risk factor for chilhood leukemia - a nationwide register-based case-control study from Denmark, Sweden, Norway and Iceland. J Natl Cancer Inst 2004; 96: 1549-1556.

12 McNally RJ, Eden TO. An infectious aetiology for childhood acute leukaemia: a review of the evidence. Br J Haematol 2004; 127: 243-263.

13 Taylor GM, Dearden S, Ravetto P, Ayres M, Watson P, Hussain A et al. Genetic susceptibility to childhood common acute lymphoblastic leukaemia is associated with polymorphic peptide-binding pocket profiles in HLA-DPB1*0201. Hum Mol Genet 2002; 11: 1585-1597.

14 Schmiegelow K, Garred P, Lausen B, Andreassen B, Petersen BL, Madsen HO. Increased frequency of mannose-binding lectin insufficiency among children with acute lymphoblastic leukemia. Blood 2002; 100: 3757-3760.

15 Wiemels JL, Smith RN, Taylor GM, Eden OB, Alexander FE, Greaves MF. Methylenetetrahydrofolate reductase (MTHFR) polymorphisms and risk of molecularly defined subtypes of childhood acute leukemia. Proc Natl Acad Sci USA 2001; 98: 4004-4009.

16 Miller RW. Deaths from childhood leukemia and solid tumors among twins and other sibs in the United States, 1960-67. J Natl Cancer Inst 1971; 46: 203-209.

17 Draper GJ, Sanders BM, Lennox EL, Brownbill PA. Patterns of childhood cancer among siblings. BrJ Cancer 1996; 74: 152-158.

18 Xiaoyan X, Hanping C. Fetal nucleated red blood cells in maternal peripheral blood and gestational age. Int J Gynaecol Obstet 2004; 87: 143-144.

19 Lee LA. Transient autoimmunity related to maternal autoantibodies: neonatal lupus. Autoimmun Rev 2005; 4: 207-213.

20 Pui CH, Schrappe M, Masera G, Nachman J, Gadner H, Eden OB et al. Ponte di Legno Working Group: statement on the right of children with leukemia to have full access to essential treatment and report on the Sixth International Childhood Acute Lymphoblastic Leukemia Workshop. Leukemia 2004; 18: 1043-1053. 